\title{
A Comparison of Methods for Computer Taxonomy
}

\author{
BY R. J. BEERS, JUNE FISHER, SALLY MEGRAW AND \\ W. R. LOCKHART \\ Department of Bacteriology, Iowa State University, \\ Ames, Iowa, U.S.A.
}

(Received 6 November 1961)

\begin{abstract}
SUMMARY
The systematic relationships among 54 strains of bacteria, representing principally the genera Achromobacter, Aerobacter, Alcaligenes, Escherichia, Mima, Pseudomonas, Serratia and Streptococcus, were examined by computer methods. Seventy-one properties of these organisms were determined, and the resulting data scored in different ways (according to various proposed techniques) before being submitted to an appropriate computer program for calculation of similarity $(\mathrm{S})$ values. These comparative studies indicated that better division of organisms into mutually similar groups can be achieved when data about properties which may have several alternative expressions are handled in the manner proposed by Beers \& Lockhart (1962). The number of comparisons which contribute to individual similarity values should be held constant by adequate treatment of quantitative data and by adoption of scoring methods which permit comparisons between 'negative' properties. It may be useful to employ distance $\left(D=\log _{2} 1 / S\right)$ rather than similarity as the primary measure of relationships among groups of organisms.
\end{abstract}

\section{INT'RODUCTION}

A number of modifications in computer techniques for the analysis of taxonomic data according to the general principles propounded by Sneath $(1957 a, b)$ were suggested by Beers \& Lockhart (1962). The present paper reports the results of some comparisons of the various proposed methodologies.

\section{METHODS}

The microbial cultures used in this study are listed below. Reference strains obtained from the American Type Culture Collection and the Iowa State University culture collection are designated by the symbols ATCC and ISU, respectively. Cultures 23, 24, 32 and 42 were obtained from the Culture Collection of Entomogenous Bacteria, Prague, Czechoslovakia, through the courtesy of Dr O. Lysenko. Representatives of the tribe Mimeae (cultures 1-5) were secured from Dr Sylvia Cary of the Walter Reed Army Hospital, Washington, D.C. Culture 25 was provided by Dr G. E. Bucher, Entomology Laboratory, Ontario, Canada. The remaining organisms were isolated in this laboratory (Raun, Lockhart \& Beers, 1959) from apparently diseased larvae of the European corn borer Ostrinia nubilalis (Hübner). These strains (designated as 'isolates') were subjected to appropriate diagnostic tests and found to conform to the descriptions of the indicated species in Bergey's 


\section{R. J. Beers, J. Fisher, S. Megraw and W. R. Lockhart}

\section{Table 1. Features employed in computer analyses of relationships among the test organisms}

* Asterisk indicates all features assigned to a property were scored ' $O$ ' (no comparison) if the preceding property was negative for a given organism.

No. of alternative features assigned for computation no.:

\section{Property}

\begin{tabular}{|c|c|c|}
\hline$\stackrel{\text { I }}{\text { (Fig. 1) }}$ & $\underset{\text { (Fig. 2) }}{\text { II }}$ & $\underset{\text { (Fig. 3) }}{\text { III }}$ \\
\hline
\end{tabular}

1. Cell morphology

2. Motility

3. Colony form

4. Colony edge

5. Smooth colonies

6. Opaque colonies

7. Granular colonies

8. Raised colonies

9. Odour in broth

10. Type of odour

11. Sediment in broth

12. Type of sediment

13. Turbidity in broth

14. Amount of turbidity

15. Pigment production

16. Colour of pigment

17. Pigment diffusible

18. Ammonia production

19. Haemolytic activity

20. Type of haemolysis

21. Gelatin liquefied

22. Starch hydrolysed

23. Casein hydrolysed

24. Hippuric acid hydrolysed

25. Oxidase produced

26. Catalase produced

27. Amount of catalase

28. Plasma jelled

29. Indole produced

30. Methyl red test

31. Voges-Proskauer test

32. Nitrate reduced

33. Acid from glucose

34. Gas from glucose

(Fig. 1) (Fig. 2) (Fig. 3)

Acid from:

35. Fructose

36. Galactose

37. Xylose

38. Arabinose

39. Maltose

40. Sucrose

41. Lactose

42. Glycerol

43. Mannitol

44. Sorbitol

45. Salicin

46. Litmus milk acid

47. Litmus reduced

48. Milk coagulated

49. Milk peptonized

\begin{tabular}{|c|c|c|}
\hline 3 & 3 & 3 \\
\hline 1 & 2 & 2 \\
\hline 3 & 3 & $\mathbf{3}$ \\
\hline 4 & 4 & 4 \\
\hline 1 & 2 & 2 \\
\hline 1 & 2 & 2 \\
\hline 1 & 2 & $\mathbf{2}$ \\
\hline 1 & 2 & 2 \\
\hline 1 & 2 & 5 \\
\hline $4^{*}$ & 4* & \\
\hline 1 & 2 & 4 \\
\hline $3^{*}$ & $3^{*}$ & -4 \\
\hline 1 & 2 & $A$ \\
\hline 3* & $3^{*}$ & 4 \\
\hline 1 & 2 & \\
\hline $4^{*}$ & 4* & \\
\hline $1 *$ & $2^{*}$ & Omitted \\
\hline 1 & 2 & 2 \\
\hline 1 & 2 & 4 \\
\hline $\mathbf{3}^{*}$ & 3* $^{*}$ & 4 \\
\hline 1 & 2 & 2 \\
\hline 1 & 2 & 2 \\
\hline 1 & 2 & 2 \\
\hline 1 & 2 & 2 \\
\hline 1 & 2 & 2 \\
\hline 1 & 2 & 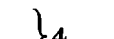 \\
\hline $3 *$ & $3^{*}$ & \\
\hline 1 & 2 & 2 \\
\hline 1 & 2 & 2 \\
\hline 1 & 2 & 2 \\
\hline 1 & 2 & 2 \\
\hline 1 & 2 & 2 \\
\hline 1 & 2 & 2 \\
\hline $1^{*}$ & $2^{*}$ & Omitted \\
\hline
\end{tabular}


Table 1 (cont.)

\begin{tabular}{|c|c|c|c|}
\hline \multirow[b]{2}{*}{ Property } & \multicolumn{3}{|c|}{$\begin{array}{l}\text { No. of alternative features } \\
\text { assigned for computation no.: }\end{array}$} \\
\hline & $\begin{array}{c}\text { I } \\
\text { (Fig. 1) }\end{array}$ & $\begin{array}{c}\text { II } \\
\text { (Fig. 2) }\end{array}$ & $\begin{array}{c}\text { III } \\
\text { (Fig. 3) }\end{array}$ \\
\hline $\begin{array}{l}\text { llurite } \\
\text { cite resistance }\end{array}$ & $\begin{array}{l}1 \\
2^{*}\end{array}$ & $\begin{array}{l}2 \\
2^{*}\end{array}$ & 3 \\
\hline ystal violet & 1 & 2 & \\
\hline al violet resistance & $2^{*}$ & $2^{*}$ & \\
\hline ethylene blue & 1 & 2 & 2 \\
\hline nicillin & 1 & 2 & \\
\hline illin resistance & 3* & $3^{*}$ & -4 \\
\hline ure to $56^{\circ}$ & 1 & 2 & \\
\hline ance to $56^{\circ}$ & 2* & $2^{*}$ & 3 \\
\hline & 1 & 2 & \\
\hline ance to $\mathrm{N}$ & $3^{*}$ & $3^{*}$ & \}$^{4}$ \\
\hline
\end{tabular}

Utilized as sole carbon source:

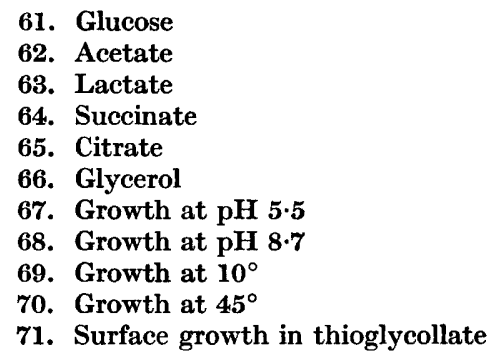

Manual (1957). A name or epithet enclosed in parentheses indicates that the isolate in question did not agree with any description listed in Bergey's Manual, but was judged to resemble most closely the organism designated.

The test organisms were:

1, Mima polymorpha, WR 26; 2, M. polymorpha, WR 45; 3, M. polymorpha, WR $980 ; 4, M$. polymorpha, WR 1957.

5, Herellea sp., WR 393.

6, Alcaligenes faecalis, ISU 2E2; 7, A. faecalis, isolate 2C-454.

8, (Vibrio curveatus), isolate 50C.

9, Achromobacter sp. (delmarvae), isolate 2C442A; 10, Achromobacter sp. (eurydice), isolate $3 \mathrm{C} 442 \mathrm{~A}$.

11, Pseudomonas reptilivora, isolate $\mathrm{B} 2 \mathrm{C} 437 ; 12, P$. reptilivora, isolate $\mathrm{I} 2 \mathrm{C} 437$; $13, P$. reptilivora, isolate $50 \mathrm{~B} ; 14, P$. boreopolis, isolate $1 \mathrm{C} 447 ; 15, P$. boreopolis, isolate $2 \mathrm{C} 447 ; 16, P$. boreopolis, isolate $1 \mathrm{C} 450 ; 17, P$. boreopolis, isolate $3 \mathrm{C} 450$.

18, Escherichia coli, ATCC 4157 (NCTC 86); 19, E. coli, ISU 2B5; 20, E. coli, ISU 4157; 21, E. coli, ISU 2B6; 22, E. coli, isolate 18C.

23, Aerobacter cloacae, CCEB 160; 24, A. cloacae, CCEB 161; 25, A. cloacae, Lister $2163 ; 26, A$. aerogenes, isolate $1 \mathrm{C3A} ; 27, A$. aerogenes, isolate $11 ; 28,($ Aerobacter sp.), isolate 1C495; 29, (Aerobacter sp.), isolate $2 \mathrm{C} 495$.

30, Proteus vulgaris, ATCC 6896 (NCTC 4636).

31, Serratia marcescens, ATCC 274. (NCTC 1377); 32, S. marcescens, CCEB 006;

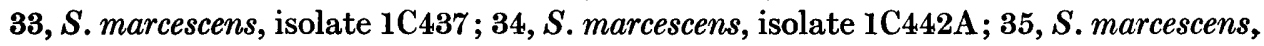




\section{R. J. Beers, J. Fisher, S. Megraw and W. R. Lockhart}

isolate $2 \mathrm{C} 81 ; 36, S$. marcescens, isolate $1 \mathrm{C} 81 ; 37, S$. marcescens, isolate 1C454; $38, S$. marcescens, isolate $1 \mathrm{C} 462 ; 39, S$. marcescens, isolate $3 \mathrm{C} 462 ; 40, S$. marcescens,

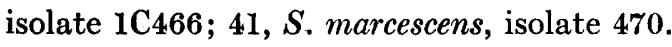

42, Streptococcus faecalis, CCEB 079; 43, S. faecalis, isolate 1C237;44, S. faecalis, isolate 2C237; 45, S. faecalis var. liquifaciens, isolate $3 \mathrm{C} 454 ; 46, S$. faecalis var. liquifaciens, isolate $2 \mathrm{C} 462 ; 47, S$. faecalis var. liquifaciens, isolate $2 \mathrm{C} 461 ; 48$, $S$. faecalis var. liquifaciens, isolate $2 \mathrm{C450} ; 49, S$. faecalis var. liquifaciens, isolate $1 \mathrm{C} 461 ; 50, S$. mitis, isolate $\mathrm{B1C437}$; $51, S$. mitis, isolate $\mathrm{B3C437} ; 52, S$. mitis, isolate $2 \mathrm{C3A} ; 53, S$. mitis, isolate $406 \mathrm{~A} ; 54, S$. mitis, isolate $2 \mathrm{C} 466$.

The properties used to determine similarities among these strains are listed in Table 1; all diagnostic tests were performed by conventional methods (Society of American Bacteriologists, 1957). Results of each test were scored as positive (t), negative ( - ), or 'no comparison' $(O)$ according to various scoring conventions. Similarity $(S)$ values for pairs of strains were calculated by means of the expression

$$
S=\frac{n_{s}}{n_{s}+n_{d}},
$$

where $n_{s}$ is the number of features positive for both organisms and $n_{d}$ is the number of features positive for one but not the other. Features negative for both, or any combination containing a zero, were omitted from the calculation.

Table 1 shows the number of alternatives used to designate each property when the scoring methods proposed by Beers \& Lockhart (1962) were used. Property number 2 (motility), for example, was assigned a single feature for computation $\mathbf{I}$, but was assigned two alternative features for computations II and III:
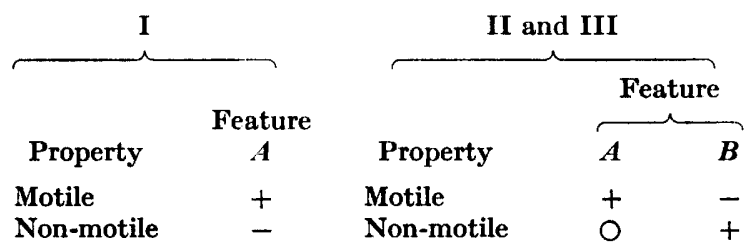

The first property, cell morphology, was scored in each case as three alternative features:

$\begin{array}{llcl}\text { Property } & \overbrace{\boldsymbol{A}}^{\text {B }} & \boldsymbol{B} \\ \text { Rods } & + & - & - \\ \text { Cocei } & \circ & + & - \\ \text { Pleomorphic } & \circ & 0 & +\end{array}$

Certain quantitative data were handled in a different manner in each computation. For example, properties 26 and 27 , having to do with catalase production, were scored as follows:

\section{Property}

Produces no detectable catalase Slight catalase production Moderate catalase production Pronounced catalase production

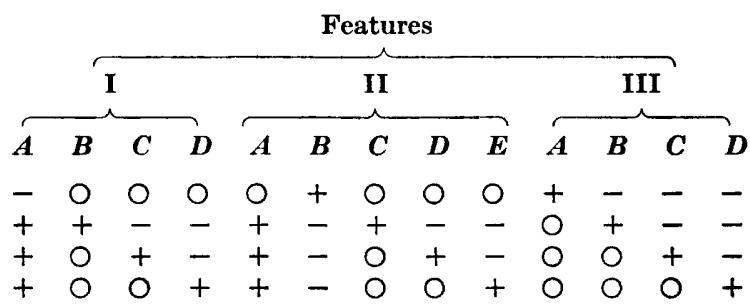


For computation I, feature $A$ was assigned to property 26 and features $B, C$ and $D$ to property 27. For II, features $A$ and $B$ were assigned to property 26 (scored as two alternatives) and features $C, D$ and $E$ to property 27 (scored as three alternatives). For computation III, properties 26 and 27 were scored together as four alternatives.

Although the calculation of $S$ was always the same, use of different scoring conventions assured that in some cases properties 'negative' for both organisms contributed to the value of $n_{s}$. We are using the term feature here in a slightly different context than did Sneath $(1957 b)$. Characteristics of organisms are referred to as properties. Features are used in scoring; a given property may be assigned one, two or more features, according to the scoring method in use.

$S$ values for each pair of strains were calculated by means of the Cyclone digital computer of Iowa State University, and the strains arranged into groups at various similarity levels by machine sorting followed by visual inspection of the data. Machine programs for computing $S$ values and sorting of strains were substantially the same as those employed by Sneath $(1957 b)$.

\section{RESULTS}

In an initial attempt to determine the relationships among the strains listed in Table 1, scoring was done entirely by the method of Sneath $(1957 b)$; that is, each property was scored as either 'positive' or 'negative', quantitative data were scored in accordance with his method $C$ (Sneath, 1957 b), and characteristics which could have more than two alternative expressions were scored as a series of separate features. Cell morphology, for example, was scored as follows:

\begin{tabular}{|c|c|c|c|}
\hline \multirow[b]{2}{*}{ Property } & \multicolumn{3}{|c|}{ Features } \\
\hline & $A$ & $B$ & $C$ \\
\hline Rods & + & - & - \\
\hline Cocci & - & + & - \\
\hline Pleomorphic & - & - & + \\
\hline
\end{tabular}

When strains were sorted into groups on the basis of $S$ values obtained in this manner, results somewhat similar to those shown in Fig. 1 were obtained. Intragroup similarities were in general quite low, and demarcations between groups were not at all clear. It was necessary, in order to achieve groupings which seemed sensible, to be quite arbitrary in deciding whether certain strains should be assigned to one or another group to which they were almost equally similar (or, rather, dissimilar).

The data were then rescored, treating certain quantitative properties, and those involving three or more alternative expressions, in the manner proposed by Beers $\&$ Lockhart (1962); the results obtained are shown in Fig. 1. In this and subsequent figures, horizontal distances indicate degrees of similarity and the horizontal position of any strain in the block diagrams indicates that it is similar to all other strains in that group at the level shown. Vertical positions of strains within a particular block (i.e. group) are of no special significance. Strain numbers correspond to those listed earlier.

The streptococci (strains 42-54) are not included in Fig. 1. In all the computer 


\section{R. J. Beers, J. Fisher, S. Megraw and W. R. Lockhart}

analyses performed, these organisms were clearly separated from all other strains, forming a homogeneous group with relatively high intragroup similarity and consisting of two subgroups. Although it is significant that the presence of these data did not interfere with sorting and that the streptococci could be readily separated, the actual values obtained are of no great interest and are omitted from the figures for the sake of simplicity. In computation I, the streptococci formed two subgroups with mean $S$ values of about $0 \cdot 85$, which fused into a single group at $S=0.50$. There was no relationship with any other group at $S$ values above $\mathbf{0 \cdot 3 0}$.

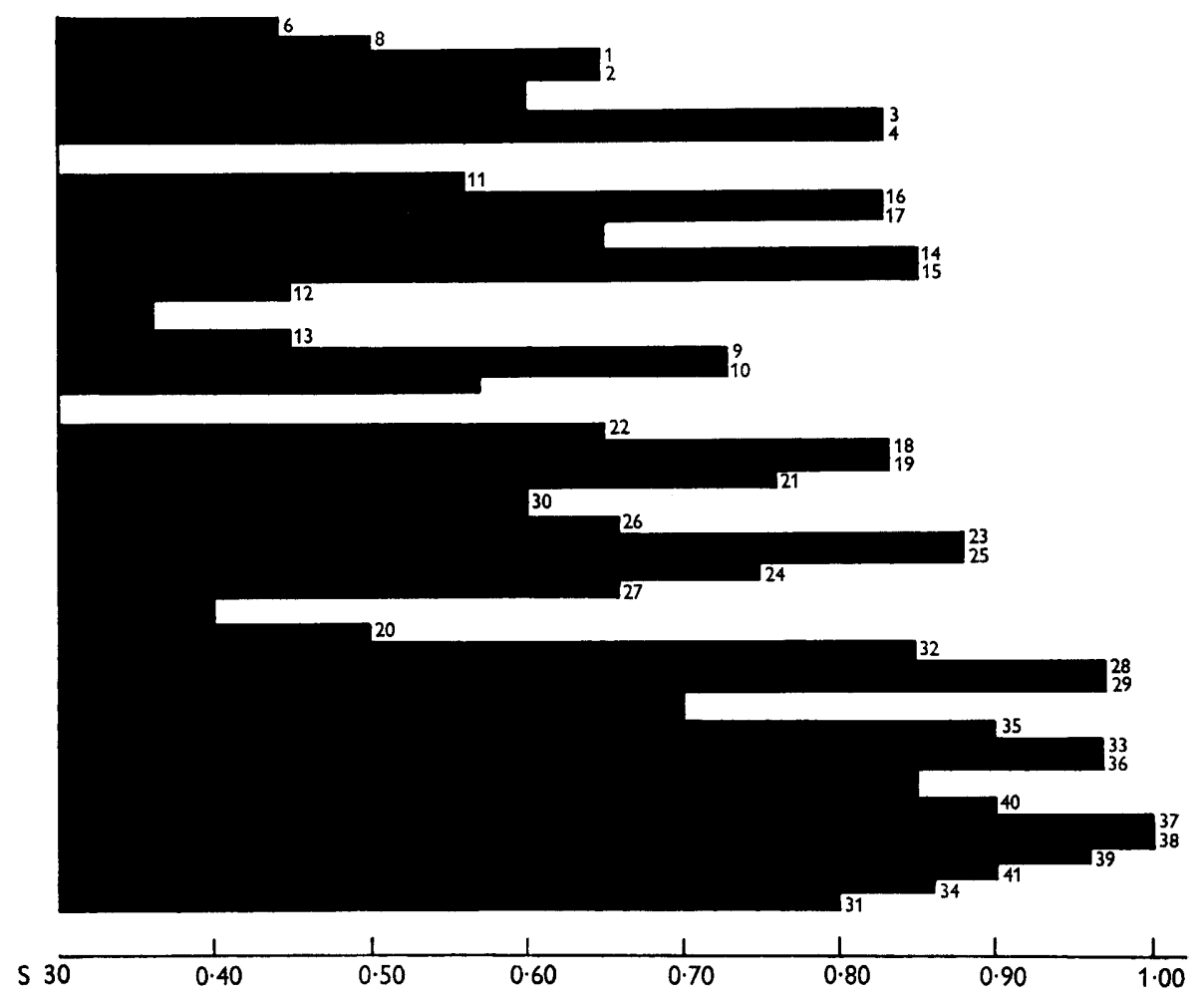

Fig. 1. Similarity relationships among the test organisms as revealed by scoring method I (Table 1).

Although the data summarized in Fig. 1 show in general the groupings one might expect, $S$ values are quite low, with no very high similarities except those between isolated pairs of strains. Intergroup similarities (even between tribes of enterobacteria) are extremely low or not detectable at all. Further, several strains appear to have found their way into the wrong groups altogether. Thus the two strains of Alcaligenes faecalis (6 and 7) appear in different groups, one of the Pseudomonas (culture 13) is in the wrong group, and one strain of Escherichia coli (20) joins the Serratia group. The single strain of Herellea (5), which is presumed to be closely related to Mima polymorpha, does not join this or any other group at any level above $S=0 \cdot 30$. The presence of culture 8 among the Mima, and cultures 28 and 29 in a subgroup with the 'entomogenous' strain of Serratia marcescens (culture 32), are apparently valid relationships, as will be seen later. 
When these same data were scored by a method which permits two strains 'negative' for a given property to be considered similar (Beers \& Lockhart, 1962), rather different results were obtained. Table 1 indicates the method of scoring (II) used to obtain the groupings shown in Fig. 2. Measured overall, similarities are greater in this case, and groups as well as pairs of strains are now detected at a relatively high similarity level. The two Alcaligenes strains (cultures 6 and 7) now appear in the same group, and the Herellea (culture 5) is in the Mima-Alcaligenes group, where one presumes it should be. Culture 13, along with the two Achromobacter (cultures 9 and 10), has joined the Pseudomonas. However, three strains

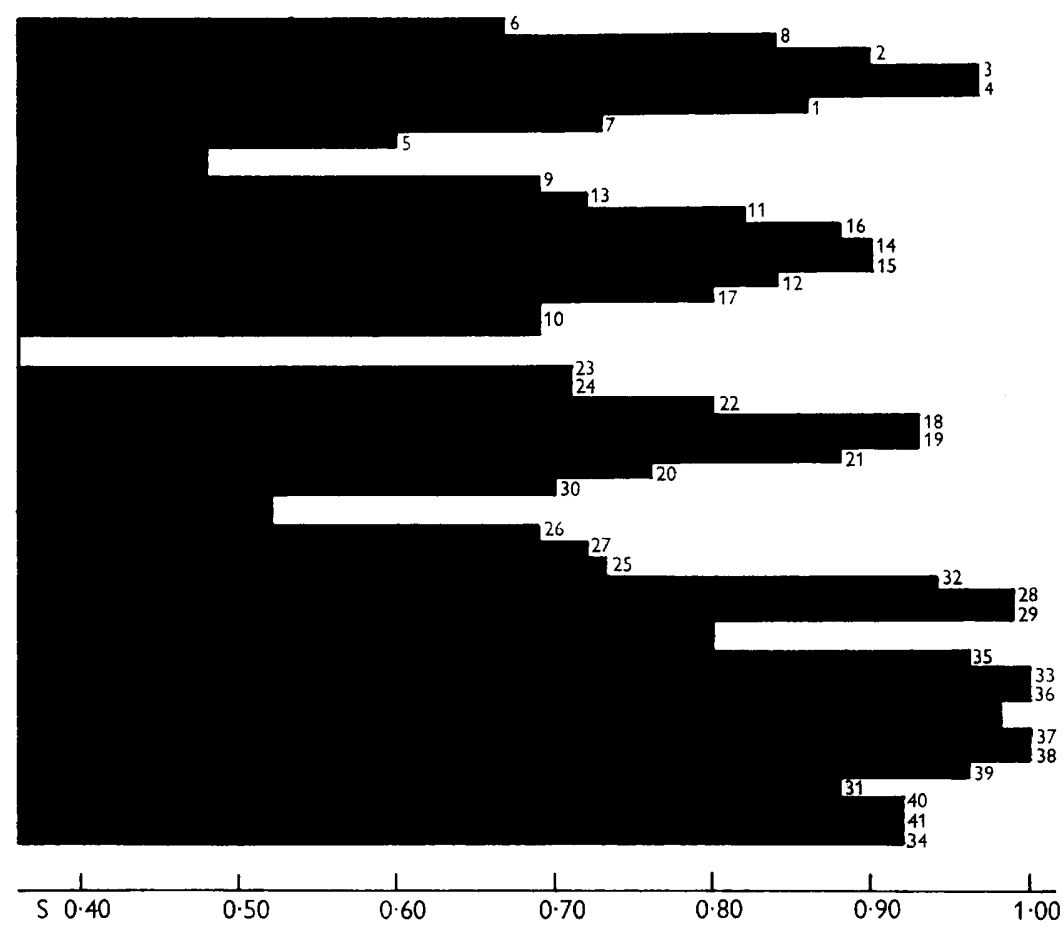

Fig. 2. Similarity relationships among the test organisms as revealed by scoring method II (Table 1).

of Aerobacter (cultures 25, 26 and 27) appear more like Serratia than EscherichiaAerobacter-Proteus, which now have fused into a single group. Minor differences also occur in the subgroupings among the strains of Serratia (cultures 31-41). The streptococci (cultures 42-54) are omitted from Fig. 2; they formed two subgroups with mean $S$ values of about 0.90 , fusing into a single group at $S=0 \cdot 72$. There was no relationship to other groups in Fig. 2 at any $S$ value above $0 \cdot 30$.

Although the results obtained by method II appear superior in some respects to those shown in Fig. 1, they still leave much to be desired. Even though 'negative' comparisons are used, the total number of features involved in computation of $S$ is not constant (and is thus a source of inconsistency) unless certain classes of quantitative data are handled in a special manner (Beers \& Lockhart, 1962). As indicated in Table 1, the coding system was then further modified (III) so that no property 


\section{R. J. Beers, J. Fisher, S. Megraw and W. R. Lockhart}

would be excluded because of the result of another test. In properties 26 and 27 , for example, failure of a strain to produce detectable amounts of catalase was merely considered one of four possible alternative expressions of its 'catalase property', and any strain (regardless of its catalase activity) scored only a single similarity or a single difference in comparison with any other strain.

The groupings obtained as a result of coding procedure III are shown in Fig. 3. Once again, similarity levels are relatively high, and involve groups rather than pairs of strains. In this instance, however, no strain appears to have been misplaced,

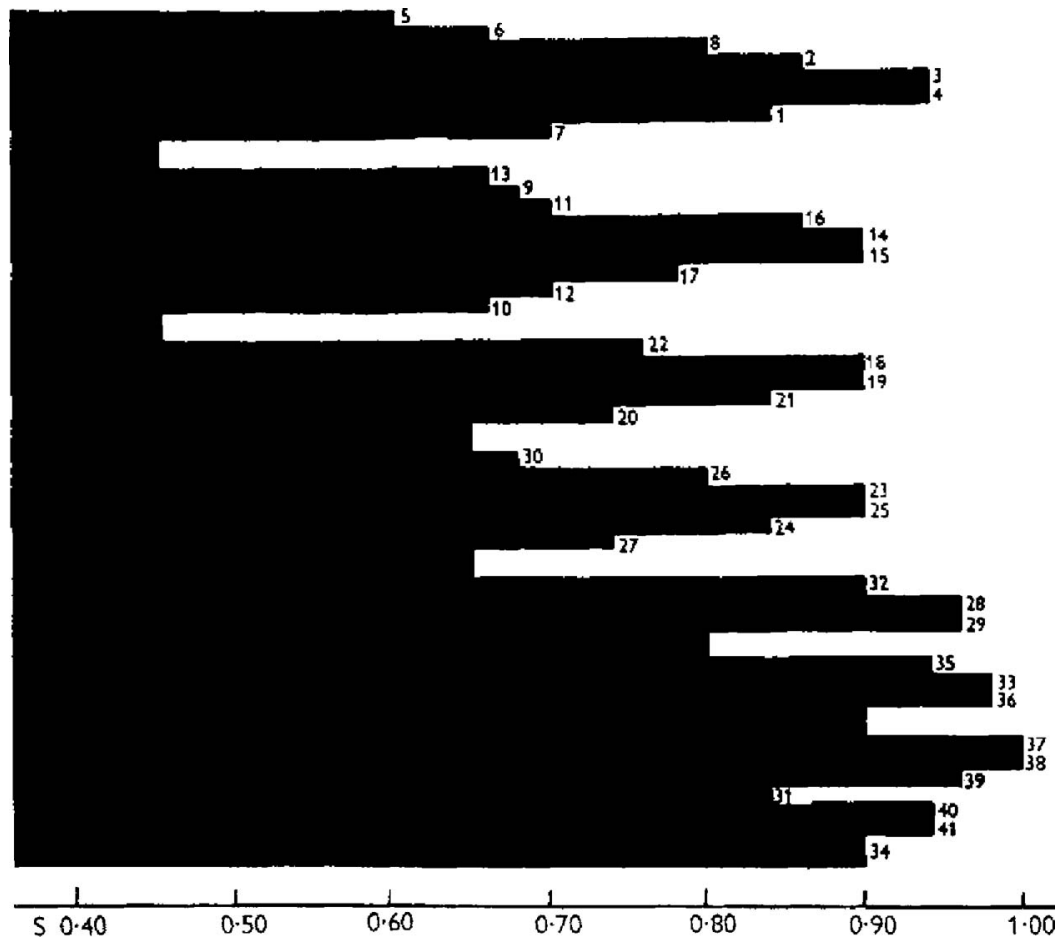

Fig. 3. Similarity relationships among the test organisms as revealed by scoring method III (Table 1).

and intergroup relationships are delineated rather well. 'The three principal groupsMimeae-Alcaligenes, Pseudomonas-Achromobacter, and enterobacteria-fuse together at $S=0 \cdot 45$. Three subgroups of enterobacteria-Escherichia, Aerobacter and Serratia-are well defined at $S=0.65$, with the single strain of Proteus (culture 30) joining in at about the same level. Serratia in turn divides into subgroups at a still higher level of similarity. The streptococci, again omitted from this diagram, consisted of the same two subgroups as before (mean similarity $=0.90$ ), fusing into a single group at $S=0.76$. Once again the streptococei did not fuse with any group shown in Fig. 3 at $S$ values above 0.80 . The three isolates which could not be identified (cultures 8,28 and 29 ) remained associated with the same groups as before.

It was suggested by Beers \& Iockhart (1962) that distance $\left(D=\log _{2} 1 / S\right)$ rather than similarity might well be adopted as the primary measure of relationships 
among organisms. The data from Fig. 3 were converted from $S$ to $D$ values; the resulting diagram is shown in Fig. 4. Use of $D$ tends to make groups appear more homogeneous, by decreasing intragroup differences at high similarities and accentuating the intergroup differences at lower similarities. The distance parameter is only a means of expressing results, of course. Figure 4, being derived from Fig. 3 , contains no information which was not already present in the latter.

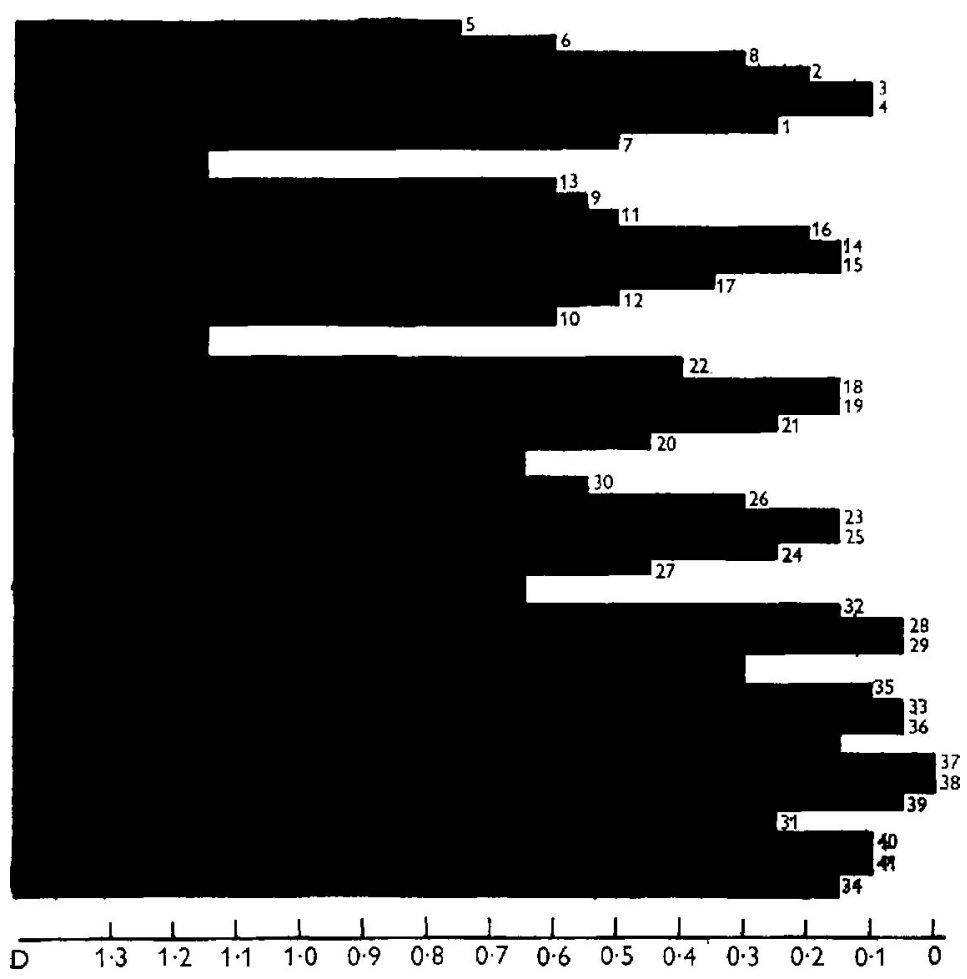

Fig. 4. Relationships among the test organisms, depicted in terms of distance $\left(D=\log _{2} 1 / S\right)$ rather than similarity. Scoring method III was used.

\section{DISCUSSION}

It appears essential that the scoring convention of Beers \& Lockhart (1962) be used for properties wherein there is no clear-cut 'negative' response, or where more than two alternatives exist. This procedure was used even in Fig. 1, where Sneath's original scoring methods were otherwise followed meticulously. From this startingpoint, it is not difficult to extend the same reasoning to all tests, and consider that at least two alternative results (neither necessarily 'negative') exist for any property of an organism which one might determine. The data in Fig. 2, and the results of Hill et al. (1961) would indicate that this procedure is both valid and desirable. In the present experiments it must be admitted that some of the differences between Figs. 1 and 2 are rather minor ones. The fact that generally higher similarity values may be obtained is not sufficient in itself to justify use of the proposed scoring system. 


\section{R. J. Beers, J. Fisher, S. Megraw and W. R. Lockhart}

It is significant, however, that relatively homogeneous groups of strains, rather than merely isolated pairs of organisms, may thus be distinguished at high similarity levels. This fact considerably simplifies the problem of sorting an array of organisms into mutually similar groups, once $S$ values have been computed. Arranging a random table of $S$ values into groups of similar organisms presents numerous pitfalls. Ideally the computer is directed to sort out all groups of organisms in which each strain is similar to every other strain at a given $S$-level. In practice, a group may exist composed of, let us say, strains $\mathbf{A}, \mathrm{C}$ and $\mathrm{D}$. It is quite possible, however, that at a given level of $S$ strain B may be similar to strain A, though not to strains $\mathrm{C}$ and $\mathrm{D}$. The computer would designate a group $A, B$, then reject strains $\mathrm{C}$ and $\mathrm{D}$ because they were not similar to both organisms already in the 'group'. The group A, C, D would be found in this example if the machine were directed to conduct the search backward, beginning with strain $D$. In a larger array of organisms, however, where two or more such 'intermediate' strains might show coincidental similarities to some members of a rather large group, such accidents could be avoided only by having the machine conduct a separate search for every possible order in which the strains could be arranged. For an array of 100 organisms, then, it would be necessary to conduct 100 factorial (about $10^{158}$ ) searches, a procedure which would consume infinite amounts of expensive machine time and produce tremendous quantities of duplicate information.

Fortunately, there should exist few individuals like 'strain $B$ ' in the above example. If there actually were in nature a continuous spectrum of such organisms intermediate between groups, it would be quite hopeless to attempt to classify micro-organisms by any means. If we are not to abandon systematics altogether, therefore, we must assume that the apparent occurrence of large numbers of such cryptic individuals results from insufficient data or from inadequate methods of computation.

It is obvious that if an extremely small number of features were used for computation of $S$ values, the 'groups' which would appear at various similarity levels would be almost entirely a matter of chance. The low similarity level within groups when certain coding systems are used seems to result from the fact that some $S$ values are, indeed, based on an insufficient number of features. That is, the strains which appear to be intermediate between existing groups are those which give a large proportion of 'negative' responses to the diagnostic tests employed. The information concerning such results may simply be lost, together with any quantitative data which are available only if certain other properties are positive. As a result, some $S$ values may be based in fact on a ridiculously small number of features.

As pointed out by Beers \& Lockhart (1962), unbiased selection of an adequately large number of tests which would give approximately equal numbers of positive and negative responses for any appreciable variety of organisms is almost impossible in practice. There would appear to be no alternative but to adopt some scoring method which ensures that the same number of criteria of similarity will be used in computation of all $S$ values. Figure 3, showing the groupings obtained when this was done, would indicate that definitely superior results are thereby obtained. Comparison of Figs. 1-3 shows that such treatment of quantitative data, or of other properties involving more than two alternatives, is quite important. Probably the suggested scoring system should be used for any such data, even by investigators who do not 
choose to consider as similarities such things as the fact that neither of two organisms produces indole.

The use of distance $(D)$ rather than similarity $(S)$ values, illustrated in Fig. 4, perhaps offers no important advantage. In some attempts to diagram the threedimensional relationships among these strains, however, we found it necessary to use $\mathrm{D}$. There at least are sound theoretical reasons for adopting this parameter, and calculation of $D$ rather than $S$ would not be difficult (Beers \& Lockhart, 1962). It should be pointed out also that Fig. 4 was obtained simply by converting the $S$ values from Fig. 3. If $D$ values were calculated directly, they would provide a new logical basis for sorting organisms into groups. Intervals on a similarity scale are different from those on the logarithmic $D$ scale. For example, all similarity levels above $S=0.93$ would be included in the groups formed within the interval between $D=0.0$ and $D=0.1$, while the distance within the interval $D=0.9$ to $D=1.0$ would include only the similarity levels between $S=0.50$ and $S=0.54$. Thus for the purpose of sorting organisms into groups, little significance would be attributed to minor differences in similarity at such high levels as $S=0 \cdot 80-1 \cdot 00$ (i.e. subgroups within species; see Figs. 3 and 4), whereas intergroup differences at low levels of similarity ( $S=\mathbf{0 . 5 0}$ or less) would receive added emphasis. Delineation of groups on this basis would perhaps be more valid than the use of similarity values.

Nothing particularly novel or surprising with regard to the relationships among these organisms is to be found in the data presented here. It was our purpose to test and compare various computer techniques rather than to develop new knowledge of the systematics of these particular organisms. It should be pointed out, however, that a suspected relationship between the tribe Mimeae and certain insect pathogens (Beers, Lockhart \& Raun, 1959) was not confirmed. Representatives of the Mimeae (cultures 1-5) showed no appreciable similarity either to authentic cultures of Coccobacillus acridiorum (= Aerobacter cloacae, cultures 23, 24 and 25) and Bacillus noctuarum ( = Serratia marcescens, culture 32), or to any of our isolates. Some of the subgroups found among the various strains of Serratia marcescens and among the streptococci (the latter are omitted from the figures) appear to be significant in relation to insect pathogenicity, but such matters are not to the present point and are better discussed in a more appropriate place. Some of our reference strains (cultures 18, 30 and 31) were used in an early survey by Sneath \& Cowan (1958). It would appear desirable to include such strains as 'reference points' in studies like these whenever possible, in order to facilitate correlation of the results obtained by different investigators.

Journal paper no. J-4213 of the Iowa Agricultural and Home Economics Experiment Station, Ames, Iowa. Project No. 1509. One of us (J. F.) was an undergraduate research participant, National Science Foundation (U.S.).

\section{REFERENCES}

BeErs, R. J. \& Lockhart, W. R. (1962). Experimental methods in computer taxonomy. J. gen. Microbiol. 28, 633.

Beers, R. J., Lockhart, W. R. \& Raun, E. S. (1959). Some characteristics of bacteria isolated from diseased larvae of the European corn borer. Proc. Iozea Acad. Sci. 66, 504.

Bergey's Manual of Determinative Bacteriology. Ed. R. S. Breed, E. G. D. Murray \& N. R. Smith (1957). Baltimore, U.S.A.: Williams and Wilkins Co. 


\section{R. J. Beers, J. Fisher, S. Megraw and W. R. Lockhart}

Hill, L. R., Turri, M., Gilardi, E. \& Silvestri, L. G. (1961). Quantitative methods in the systematics of Actinomycetales. II. G. Microbiol. 9, 56.

Raun, E. S., Lockhart, W. R. \& Beers, R. J. (1959). Microorganisms found in field specimens of diseased corn borer larvae. Proc. Iowa Acad. Sci. 66, 508.

SNeAth, P. H. A. (1957 a). Some thoughts on bacterial classification. J. gen. Microbiol. 17, 184.

SNEath, P. H. A. (1957b). The application of computers to taxonomy. J. gen. Microbiol. 17, 201.

Sneath, P. H. A. \& Cowan, S. T. (1958). An electrotaxonomic survey of bacteria. J. gen. Microbiol. 19, 551.

Society of American Bacteriologists (1957). Manual of Microbiological Methods. New York: McGraw-Hill. 